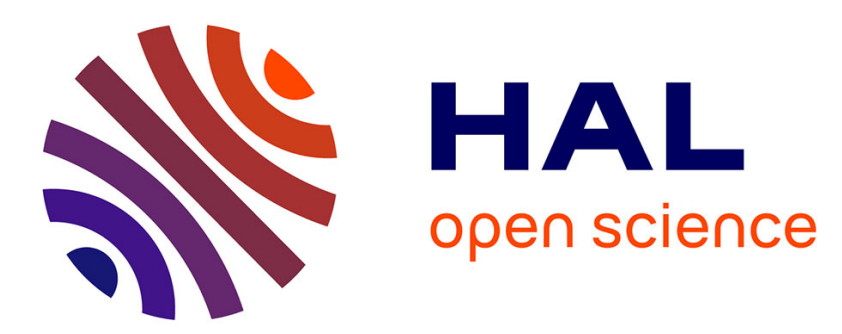

\title{
Exact Symbol Error Probability of Hybrid/Integrated Satellite-Terrestrial Cooperative Network
}

\author{
Sokchenda Sreng, Benoît Escrig, Marie-Laure Boucheret
}

\section{To cite this version:}

Sokchenda Sreng, Benoît Escrig, Marie-Laure Boucheret. Exact Symbol Error Probability of Hybrid/Integrated Satellite-Terrestrial Cooperative Network. IEEE Transactions on Wireless Communications, 2013, vol. 12 ( $\mathrm{n}^{\circ} 3$ ), pp. 1310-1319. 10.1109/TWC.2013.013013.120899 . hal-01154192

\section{HAL Id: hal-01154192 \\ https://hal.science/hal-01154192}

Submitted on 26 May 2015

HAL is a multi-disciplinary open access archive for the deposit and dissemination of scientific research documents, whether they are published or not. The documents may come from teaching and research institutions in France or abroad, or from public or private research centers.
L'archive ouverte pluridisciplinaire HAL, est destinée au dépôt et à la diffusion de documents scientifiques de niveau recherche, publiés ou non, émanant des établissements d'enseignement et de recherche français ou étrangers, des laboratoires publics ou privés. 


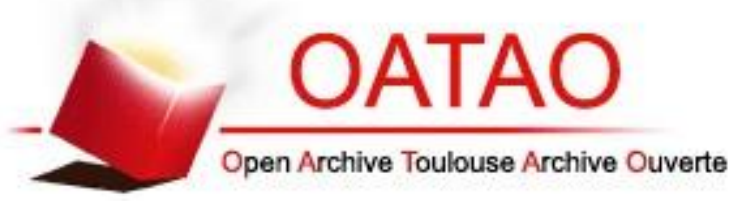

Open Archive Toulouse Archive Ouverte (OATAO)

OATAO is an open access repository that collects the work of Toulouse researchers and makes it freely available over the web where possible.

This is an author-deposited version published in: http://oatao.univ-toulouse.fr/ Eprints ID: 13084

Identification number: DOI: 10.1109/TWC.2013.013013.120899

Official URL: http://dx.doi.org/10.1109/TWC.2013.013013.120899

\section{To cite this version:}

Sreng, Sokchenda and Escrig, Benoît and Boucheret, Marie-Laure Exact Symbol Error Probability of Hybrid/Integrated Satellite-Terrestrial Cooperative Network. (2013) IEEE Transactions on Wireless Communications, vol. $12\left(\mathrm{n}^{\circ}\right.$ 3). pp. 1310-1319. ISSN 1536-1276

Any correspondence concerning this service should be sent to the repository administrator: staff-oatao@inp-toulouse.fr 


\title{
Exact Symbol Error Probability of Hybrid/Integrated Satellite-Terrestrial Cooperative Network
}

\author{
Sokchenda Sreng, Benoît Escrig, Member, IEEE, and Marie-Laure Boucheret
}

\begin{abstract}
In this paper, we study the Symbol Error Probability (SEP) performance of a hybrid/integrated satellite-terrestrial cooperative network. In particular, we focus on the case of mobile relays that forward the satellite signal to a masked mobile destination node. The Selective Decode-and-Forward (SDF) transmission scheme is implemented and only the relay nodes which can successfully decode the satellite message are selected to retransmit the signal. The destination node exploits the spatial diversity advantages by implementing a typical Maximum Ratio Combining (MRC) technique. The closed-form expressions for the exact average SEP of the arbitrary M-ary phase shift keying and M-ary quadrature amplitude modulation signaling with MRC diversity reception over independent but not necessarily identically distributed fading channels are derived using a Moment Generating Function (MGF) approach. These closed-form expressions are represented in terms of a finite sum of Lauricella hypergeometric functions. The analytical expressions show excellent agreement with the simulation results. Numerical results show that for a system using QPSK under the frequent heavy shadowed fading condition, the diversity gain of approximately $7 \mathrm{~dB}$ can be obtained at the SEP of $10^{-1}$ with respect to the direct transmission, when only one relay is used. It increases to around $12 \mathrm{~dB}$ in the case of 3 relays.
\end{abstract}

Index Terms-Symbol Error Probability (SEP), Land Mobile Satellite (LMS) channel, Mobile Satellite Systems (MSSs), hybrid/integrated satellite-terrestrial cooperative network, mobile relay, Selective Decode and Forward (SDF), Maximum Ratio Combining (MRC), Lauricella hypergeometric function, Moment Generating Function (MGF).

\section{INTRODUCTION}

$\mathbf{S}$ ATELLITE systems are used in the context of broadcasting, navigation, rescue, and disaster relief since they allow the provision of services over a wide coverage area. However, this coverage area is limited by the masking effect caused by obstacles that block the Line-Of-Sight (LOS) link between the satellite and a terrestrial user. The masking effect becomes more severe in case of low satellite elevation angles or when the user is indoor. This is the main limitation of Mobile Satellite Systems (MSSs). To tackle this problem, hybrid/integrated satellite-terrestrial cooperative systems have been proposed [1], [2].

The authors are with the University of Toulouse, IRIT Laboratory, School of ENSEEIHT, Institut National Polytechnique de Toulouse, France (e-mail: \{sokchenda.sreng, benoit.escrig, marie-laure.boucheret\} @enseeiht.fr).

Digital Object Identifier 10.1109/TWC.2013.013013.120899
The concept of the integrated satellite-terrestrial systems has been introduced in [3] with the aim of integrating the satellite with the terrestrial cellular systems for future multimedia services while hybrid systems have been proposed for satellitebased Unlimited Mobile TV systems using the Digital Video Broadcasting- Satellite to Handheld (DVB-SH) standard [4]. The main difference between hybrid and integrated systems is on whether both space and terrestrial segments use a common network and spectrum. In a hybrid network, the terrestrial gap fillers are used to relay the satellite signal independently from the satellite and do not necessarily operate in the same frequency band whereas the ground component of an integrated system is a complementary part of the satellite system and is controlled by the satellite resource and network management system. In the integrated system, the ground component uses the same portions of MMSs frequency bands as the associated satellite component.

In a hybrid/integrated satellite-terrestrial cooperative system, the mobile user can exploit the advantages of spatial diversity by receiving signals from both satellite and terrestrial components. Fixed or mobile gap-fillers are used to relay the satellite signal by implementing Amplify-and-Forward (AF), Fixed Decode-and-Forward (FDF) or Selective Decode-andForward (SDF) transmission schemes [5], [6]. In the AF transmission scheme, the relay amplifies both the source messages and the channel noise which leads to some performance degradations. In the FDF transmission scheme, all relays decode the source messages (demodulate) first and then re-encode (re-modulate) the signals before forwarding to the destination node while in the SDF transmission scheme, only the relays that can decode the source messages correctly are allowed to retransmit the signals. This SDF transmission scheme prevents the retransmission of erroneous messages to the destination node. Most of satellite broadcasting systems have been implemented using fixed gap-fillers. However, mobile gap-fillers are needed in emergency cases when the fixed infrastructure is not available. In emergency scenarios (e.g., fire, earthquake, flood and explosion), the existing terrestrial infrastructure has been destroyed. So, a hybrid satellite-terrestrial cooperative system is appropriate for updating the information. This allows the rescuers to operate efficiently and in a safe way [7], [8]. In particular, a fast and flexible implementation is needed and this could be provided by deploying mobile gap fillers (vehicle or mobile handheld).

Several cooperative scenarios for hybrid satellite-terrestrial 
systems have been proposed for this context. In [9], the delay diversity technique for a hybrid satellite-terrestrial DVB$\mathrm{SH}$ system has been studied. In this scenario, user stations receive different versions of the same signal with different delays: one signal from the satellite and other signals from terrestrial relays. No combiner is implemented. In [10], spacetime codes and rate compatible turbo codes have been implemented to achieve diversity gains and additional coding gains. Recently, hybrid satellite-terrestrial systems employing mobile gap fillers have been presented in [11], [12]. In [11], a hybrid solution based on a cooperative ad-hoc networking approach has been proposed for terrestrial links while the DVB-SH and the Next Generation Universal Mobile Satellite Telecommunications Systems (S-UMTS) standards are considered for forward (satellite broadcasting) and return (user terminal to satellite) links respectively. In [12], a two time-slot scenario has been presented. The satellite broadcasts the information to terrestrial users in a first time slot and in a second time slot, non-masked terminals are used to relay the information toward masked terminals. The Symbol Error Probability (SEP) performance has not been studied yet in [11], [12]. However, most of the previous articles on hybrid/integrated satelliteterrestrial cooperative systems have provided only the SEP performance based on the simulations [9], [10] and the SEP based on the numerical calculations [13] for the case of AF transmission schemes. The exact closed-form SEP of hybrid/integrated satellite-terrestrial cooperative systems has not been derived yet.

A Land Mobile Satellite (LMS) channel model is needed to evaluate the performance of MSSs. The most popular LMS models are Loo's model [14]-[17], Lutz's model [18], and Perez-Fontan's model [19]-[21]. In Loo's model, the amplitude of the LOS component is assumed to be lognormally distributed while the multipath interference has a Rayleigh distribution. The Lutz's model differs from the Loo's model. The latter model is a single state model whereas the Lutz's model is described by two states, good state (Rice model) and bad state (Suzuki model). The Perez-Fontan's model consists of three states, LOS, moderate shadowing and deep shadowing. Moreover, each state of the Perez-Fontan's model is described by the Loo's model. Recently, [22] has proposed a new LMS model which is an alternative to the Loo's model. The difference between the two models is that the lognormal distribution in the Loo's model is replaced by the Nakagami distribution. It has been shown that this new model provides a similar fit to the experimental data as the Loo's model but with significantly less computational burden. In the following sections, we will use this new LMS channel model to derive the average SEP of the system.

In this paper, we investigate the performance of a hybrid/integrated satellite-terrestrial cooperative system using mobile relays over independent but not necessarily identically distributed fading channels. In the first phase, the satellite broadcasts its signal to all mobile relay nodes ${ }^{1}$ and the destination node. In the second phase, the non masked mobile relay nodes forward the satellite signal to the destination node using the SDF transmission scheme. By using an approach

${ }^{1}$ The mobile relay nodes can be vehicles or mobile handhelds. based on the Moment Generating Function (MGF), the exact closed-form of the SEP is evaluated. Such closed-form solutions are highly desirable because they allow for rapid and efficient evaluation of the system performance. Furthermore, the diversity analysis is also provided. It is shown that the diversity order of $L+1$ can be obtained when the total number of relays is equal to $L$. The analytical results are then confirmed using Monte Carlo simulations.

The rest of this paper is organized as follows. The system and channel models are discussed in Section II. In Section III, the MGF and the average SEP are derived for different types of modulation schemes. The simulation and numerical results are presented in Section IV. The conclusion is finally drawn in Section V.

\section{System And Channel Models}

Consider a hybrid/integrated satellite-terrestrial cooperative system where a satellite $(s)$ transmits information to a destination node $(d)$, with the assistance of $L$ mobile relay nodes $r_{1}, r_{2}, \ldots, r_{L}$ as shown in Fig. 1. The transmission is divided into two phases. In the first phase (broadcasting phase), the satellite broadcasts its signal to the set of $L$ relay nodes and the destination node. The baseband received signal at the destination and the relay $r_{i}$ can be modeled, respectively, as

$$
\begin{aligned}
y_{s d} & =\sqrt{E_{s}} h_{s d} x+n_{s d} \\
y_{s r_{i}} & =\sqrt{E_{s}} h_{s r_{i}} x+n_{s r_{i}}
\end{aligned}
$$

where $E_{s}$ is the average transmitted energy per symbol of the satellite, $h_{s d}$ is the channel gain between the satellite and the destination, $h_{s r_{i}}$ is the channel gain between the satellite and the relay $r_{i}, x$ is the transmitted symbol with unit power, $n_{s d}$ and $n_{s r_{i}}$ are the Additive White Gaussian Noise (AWGN) of the satellite-destination link and at the satellite-relay $r_{i}$ link respectively.

We define the decoding set $C$ (with cardinality $|C|$ ) as the set of relays that can decode the satellite message correctly. The relay node is said to belong to the decoding set provided that the received Signal-to-Noise Ratio (SNR) on a channel between the source and the relay node is high enough to allow for successful decoding. We have $|C| \leq L$. In the second phase (relaying phase), only the relay nodes which belong to the set $C$ are allowed to forward the re-modulated signals to the destination node using orthogonal channels ${ }^{2}$. In the following analysis, we assume that both satellite and terrestrial links use the same modulation scheme. The baseband received signal at the destination from the relay $r_{i}$ can be modeled as

$$
y_{r_{i} d}=\left\{\begin{array}{l}
\sqrt{E_{r_{i}}} h_{r_{i} d} \widehat{x}+n_{r_{i} d}, \text { when } r_{i} \in C \\
0, \text { otherwise }
\end{array}\right.
$$

where $E_{r_{i}}$ is the average transmitted energy per symbol of the relay $r_{i}, h_{r_{i} d}$ is the channel gain between the relay $r_{i}$ and the destination, $\widehat{x}$ is the decoded symbol at the relay $r_{i}$ with unit power and $n_{r_{i} d}$ is the AWGN of the relay $r_{i}$-destination link. In SDF schemes, the estimated symbol $\widehat{x}$ is assumed to

\footnotetext{
${ }^{2}$ These orthogonal channels can be allocated to the relays using Frequency Division Multiple Access (FDMA), Time Division Multiple Access (TDMA) or Space Time Coding (STC).
} 


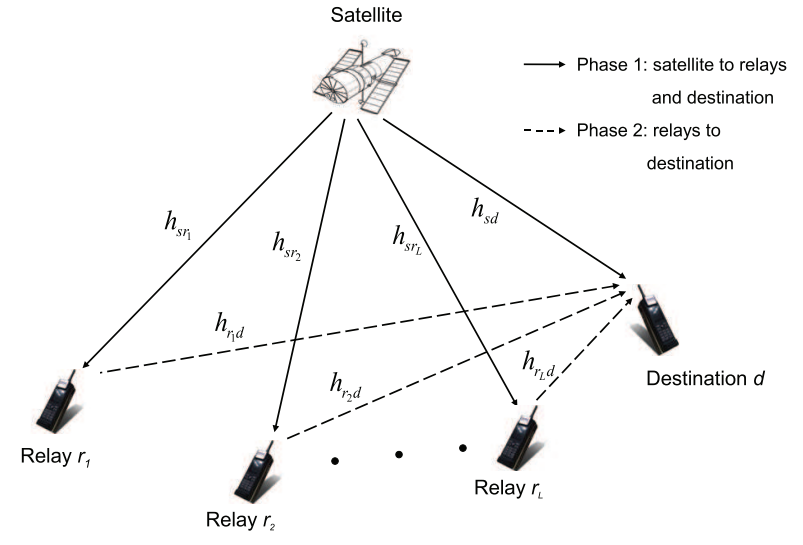

Fig. 1. Hybrid satellite-terrestrial system with $L$ relays and one destination.

be error free, i.e., $\widehat{x}=x$. This can be achieved by allowing the decoding of the satellite message only when the received SNR allows for a successful decoding or by using a Cyclic Redundancy Check (CRC). Then the destination combines the direct and the relay links signals using the Maximum Ratio Combining (MRC) technique.

We assume that the channels are frequency-flat, slow fading and independent but not necessarily identically distributed fading channels. The condition for the relays having independent fading channels is on the minimum separation distance between each relay's antenna. In general, this minimum distance is in the order of $\lambda / 2$, where $\lambda$ is the carrier signal wavelength. We also assume that the Channel State Information (CSI) is perfectly known at the receiver and not known at the transmitter ${ }^{3}$. Furthermore, we assume that the AWGN terms of all links have zero mean and equal variance $N_{0}$. The statistics of the channel models are defined as follows.

- The satellite-destination and the satellite-relays link are modeled as LMS fading channels [22]. The probability density function (PDF), $f_{\left|h_{s x}\right|^{2}}(y)$ of the power channel gain, $\left|h_{s x}\right|^{2}$, is given in [22] as

$$
\begin{array}{r}
f_{\left|h_{s x}\right|^{2}}(y)=\frac{1}{2 b_{s x}}\left(\frac{2 b_{s x} m_{s x}}{2 b_{s x} m_{s x}+\Omega_{s x}}\right)^{m_{s x}} \exp \left(-\frac{y}{2 b_{s x}}\right) \\
\times{ }_{1} F_{1}\left(m_{s x} ; 1 ; \frac{\Omega_{s x} y}{2 b_{s x}\left(2 b_{s x} m_{s x}+\Omega_{s x}\right)}\right) \text {, for } y>0
\end{array}
$$

where the second subscript $x \equiv d$ and $x \equiv r_{i}$ when we deal with the satellite to the destination and the satellite to the relay $r_{i}$ channels respectively. The parameter $\Omega_{s x}$ is the average power of the LOS component, $2 b_{s x}$ is the average power of the multipath component, and $m_{s x}$ is the Nakagami parameter ranging from 0 to $\infty$. When $m_{s x}=0$, the PDF of $\left|h_{s x}\right|$ becomes a Rayleigh PDF and when $m_{s x}=\infty$, it becomes a Rice PDF. The function ${ }_{1} F_{1}(a ; b ; z)$ is the confluent hypergeometric

\footnotetext{
${ }^{3}$ Because of the slow fading, accurate channel estimation is possible at
} receivers. function defined in [23] as

$$
{ }_{1} F_{1}(a ; b ; z)=\sum_{n=0}^{\infty} \frac{(a)_{n}}{(b)_{n}} \frac{z^{n}}{n !}
$$

where $(x)_{n}=x(x+1) \ldots(x+n-1)$.

- The $i^{t h}$ relay-destination link is modeled as a Rayleigh fading channel. The PDF, $f_{\left|h_{r_{i}}\right|^{2}}(y)$ of the power channel gain, $\left|h_{r_{i} d}\right|^{2}$, is defined in [24] as the exponential distribution

$$
f_{\left|h_{r_{i} d}\right|^{2}}(y)=\frac{1}{2 b_{r_{i} d}} \exp \left(-\frac{y}{2 b_{r_{i} d}}\right), \text { for } y>0
$$

where $2 b_{r_{i} d}$ is the average power of the multipath component of the $i^{\text {th }}$ relay-destination link.

\section{Performance AnAlysis}

In this section, we analyze the performance of the hybrid/integrated satellite-terrestrial cooperative network in terms of SEPs for general MPSK and MQAM modulation schemes. We first express the SEP of the direct link and then the exact closed-form expression of the cooperative network is derived.

\section{A. Average SEP of the Direct Link}

The instantaneous received SNR of the direct link at the receiver is given by

$$
\gamma_{s d}=\left|h_{s d}\right|^{2} \times \bar{\gamma}_{s d}
$$

where $\bar{\gamma}_{s d}=E_{s} / N_{0}$, is the average transmitted SNR per symbol of the satellite-destination link. So, the PDF of $\gamma_{s d}$ can be written as

$$
\begin{aligned}
f_{\gamma_{s d}}(y) & =\frac{1}{\bar{\gamma}_{s d}} \times f_{\left|h_{s d}\right|^{2}}\left(\frac{y}{\bar{\gamma}_{s d}}\right) \\
& =\frac{1}{2 b_{s d} \bar{\gamma}_{s d}}\left(\frac{2 b_{s d} m_{s d}}{2 b_{s d} m_{s d}+\Omega_{s d}}\right)^{m_{s d}} \exp \left(-\frac{y}{2 b_{s d} \bar{\gamma}_{s d}}\right) \\
& \times{ }_{1} F_{1}\left(m_{s d} ; 1 ; \frac{\Omega_{s d} y}{2 b_{s d} \bar{\gamma}_{s d}\left(2 b_{s d} m_{s d}+\Omega_{s d}\right)}\right), \text { for } y>0 .
\end{aligned}
$$

Hence, the MGF of $\gamma_{s d}$ can be evaluated as follows

$$
\phi_{\gamma_{s d}}(s)=\mathbb{E}\left[e^{-s y}\right]=\int_{0}^{\infty} e^{-s y} f_{\gamma_{s d}}(y) d y
$$

where $\mathbb{E}[$.$] is the mathematical expectation operation. By$ using the table of integrals in [23], $\phi_{\gamma_{s d}}(s)$ can be expressed as below

$$
\phi_{\gamma_{s d}}(s)=\frac{\left(2 b_{s d} m_{s d}\right)^{m_{s d}}\left(1+2 b_{s d} \bar{\gamma}_{s d} s\right)^{m_{s d}-1}}{\left[\left(2 b_{s d} m_{s d}+\Omega_{s d}\right)\left(1+2 b_{s d} \bar{\gamma}_{s d} s\right)-\Omega_{s d}\right]^{m_{s d}}} .
$$

Using the MGF-based analysis method [24], we will, in the following parts, derive the closed-form error rates for several modulation schemes in uncorrelated fading channels. 
1) M-ary Phase-Shift Keying (MPSK): The average SEP of the direct link for coherent MPSK signals is given by [25]

$$
\begin{array}{r}
P_{s d, M P S K}(E)=\frac{1}{\pi} \int_{0}^{\pi-\frac{\pi}{M}} \phi_{\gamma_{s d}}\left(\frac{g_{M P S K}}{\sin ^{2} \theta}\right) d \theta \\
=\underbrace{\frac{1}{\pi} \int_{0}^{\frac{\pi}{2}} \phi_{\gamma_{s d}}\left(\frac{g_{M P S K}}{\sin ^{2} \theta}\right) d \theta}_{I_{1, M P S K}^{s d}}+\underbrace{\frac{1}{\pi} \int_{\frac{\pi}{2}}^{\pi-\frac{\pi}{M}} \phi_{\gamma_{s d}}\left(\frac{g_{M P S K}}{\sin ^{2} \theta}\right) d \theta}_{I_{2, M P S K}^{s d}}
\end{array}
$$

where $g_{M P S K}=\sin ^{2}(\pi / M)$. The first integral

$$
I_{1, M P S K}^{s d}=\frac{1}{\pi} \int_{0}^{\frac{\pi}{2}} \phi_{\gamma_{s d}}\left(\frac{g_{M P S K}}{\sin ^{2} \theta}\right) d \theta
$$

can be calculated by replacing (8) into (9) and changing the variable $t=\cos ^{2}(\theta)$ and using the table of integrals in [23],

$$
\begin{aligned}
& \int_{0}^{1} t^{a-1}(1-t)^{c-a-1}(1-v t)^{-b_{1}}(1-\nu t)^{-b_{2}} d t \\
& =\frac{\Gamma(a) \Gamma(c-a)}{\Gamma(c)} F_{1}\left(a, b_{1}, b_{2} ; c ; v, \nu\right), \operatorname{Re}(c)>\operatorname{Re}(a)>0
\end{aligned}
$$

where $F_{1}\left(a, b_{1}, b_{2} ; c ; v, \nu\right)$ is the Appell hypergeometric function, $\Gamma(n)=(n-1)$ ! is the gamma function and $\operatorname{Re}($.$) denotes$ the real part. Hence, $I_{1, M P S K}^{s d}$ can be obtained as

$$
\begin{array}{r}
I_{1, M P S K}^{s d}=\frac{\left(2 b_{s d} m_{s d}\right)^{m_{s d}} G_{1, M P S K}^{m_{s d}-1}}{4 G_{2, M P S K}^{m_{s d}}} \\
\times F_{1}\left(\frac{1}{2}, 1-m_{s d}, m_{s d} ; 2 ; \frac{1}{G_{1, M P S K}}, \frac{2 b_{s d} m_{s d}}{G_{2, M P S K}}\right)
\end{array}
$$

where $G_{1, M P S K}=1+2 b_{s d} \bar{\gamma}_{s d} g_{M P S K} \quad$ and $G_{2, M P S K}=2 b_{s d} m_{s d}+2 b_{s d} \bar{\gamma}_{s d} g_{M P S K}\left(2 b_{s d} m_{s d}+\Omega_{s d}\right)$.

The second integral $I_{2, M P S K}^{s d}$ can be rewritten as

$$
I_{2, M P S K}^{s d}=\frac{1}{\pi} \int_{\frac{\pi}{M}}^{\frac{\pi}{2}} \phi_{\gamma_{s d}}\left(\frac{g_{M P S K}}{\sin ^{2} \theta}\right) d \theta .
$$

Using variable transform $t=\cos ^{2}(\theta) / \cos ^{2}(\pi / M)$, we have the closed-form for $I_{2, M P S K}^{s d}$ as shown below

$$
\begin{array}{r}
I_{2, M P S K}^{s d}=\frac{\sqrt{\omega}\left(2 b_{s d} m_{s d}\right)^{m_{s d}} G_{1, M P S K}^{m_{s d}-1}}{\pi G_{2, M P S K}^{m_{s d}}} \\
\times F_{D}^{(3)}\left(\frac{1}{2},-\frac{1}{2}, 1-m_{s d}, m_{s d} ; \frac{3}{2} ; \omega, \frac{\omega}{G_{1, M P S K}}, \frac{2 b_{s d} m_{s d} \omega}{G_{2, M P S K}}\right)
\end{array}
$$

where $\omega=1-g_{M P S K}$ and $F_{D}^{(3)}$ is the Lauricella function [26] defined as

$$
\begin{gathered}
F_{D}^{(n)}\left(a, b_{1}, \ldots, b_{n} ; c ; x_{1}, \ldots, x_{n}\right) \\
\triangleq \sum_{i_{1}, \ldots, i_{n}=0}^{+\infty} \frac{(a)_{i_{1}+\ldots+i_{n}}}{(c)_{i_{1}+\ldots+i_{n}}} \prod_{j=1}^{n}\left(b_{j}\right)_{i_{j}} \frac{x_{j}^{i_{j}}}{i_{j} !}, \max \left\{\left|x_{i}\right|\right\}_{i=1}^{n}<1 \\
=\frac{1}{B(a, c-a)} \times \int_{0}^{1} t^{a-1}(1-t)^{c-a-1} \prod_{i=1}^{n}\left(1-x_{i} t\right)^{-b_{i}} d t \\
\operatorname{Re}(c)>\operatorname{Re}(a)>0
\end{gathered}
$$

where $(a)_{i} \triangleq \Gamma(a+i) / \Gamma(a)$ is the Pochammer symbol for $i \geq 0, B(a, b) \triangleq \Gamma(a) \Gamma(b) / \Gamma(a+b)$ denotes the Beta function, and $\operatorname{Re}($.$) denotes the real part. When the number$ of variables $n$ is equal to one, $F_{D}^{(1)}$ reduces to a gaussian hypergeometric function ${ }_{2} F_{1}\left(a, b_{1} ; c ; x_{1}\right)$ and when $n$ is equal to two, $F_{D}^{(2)}$ reduces to an Appell hypergeometric function $F_{1}\left(a, b_{1}, b_{2} ; c ; x_{1}, x_{2}\right)$. So, the average SEP of the direct link, $P_{s d, M P S K}(E)$ is finally given as in equation (16).

2) M-ary Quadrature Amplitude Modulation (MQAM): $\theta$ The average SEP of the direct link for coherent MQAM signals is given by [25]

$$
\begin{aligned}
P_{s d, M Q A M}(E) & =\underbrace{\frac{4 q}{\pi} \int_{0}^{\frac{\pi}{2}} \phi_{\gamma_{s d}}\left(\frac{g_{M Q A M}}{\sin ^{2} \theta}\right) d \theta}_{I_{1, M Q A M}^{\text {sd }}} \\
& -\underbrace{\frac{4 q^{2}}{\pi} \int_{0}^{\frac{\pi}{4}} \phi_{\gamma_{s d}}\left(\frac{g_{M Q A M}}{\sin ^{2} \theta}\right) d \theta}_{I_{2, M Q A M}^{\text {sd }}}
\end{aligned}
$$

where $g_{M Q A M}=3 / 2(M-1)$ and $q=(1-1 / \sqrt{M})$. Using the same computation as in (10), the first integral

$$
I_{1, M Q A M}^{s d}=\frac{4 q}{\pi} \int_{0}^{\frac{\pi}{2}} \phi_{\gamma_{s d}}\left(\frac{g_{M Q A M}}{\sin ^{2} \theta}\right) d \theta
$$

can be obtained as

$$
\begin{array}{r}
I_{1, M Q A M}^{s d}=\frac{q\left(2 b_{s d} m_{s d}\right)^{m_{s d}} G_{1, M Q A M}^{m_{s d}-1}}{G_{2, M Q A M}^{m_{s d}}} \\
\times F_{1}\left(\frac{1}{2}, 1-m_{s d}, m_{s d} ; 2 ; \frac{1}{G_{1, M Q A M}}, \frac{2 b_{s d} m_{s d}}{G_{2, M Q A M}}\right)
\end{array}
$$

where $G_{1, M Q A M}=1+2 b_{s d} \bar{\gamma}_{s d} g_{M Q A M}$ and $G_{2, M Q A M}=2 b_{s d} m_{s d}+2 b_{s d} \bar{\gamma}_{s d} g_{M Q A M}\left(2 b_{s d} m_{s d}+\Omega_{s d}\right)$. Making the change of variable $t=1-\tan ^{2} \theta$ in the second integral

$$
I_{2, M Q A M}^{s d}=\frac{4 q^{2}}{\pi} \int_{0}^{\frac{\pi}{4}} \phi_{\gamma_{s d}}\left(\frac{g_{M Q A M}}{\sin ^{2} \theta}\right) d \theta
$$

we can find the closed-form as shown below

$$
\begin{array}{r}
I_{2, M Q A M}^{s d}=\frac{2 q^{2}\left(2 b_{s d} m_{s d}\right)^{m_{s d}} L_{1, M Q A M}^{m_{s d}-1}}{3 \pi L_{2, M Q A M}^{m_{s d}}} \\
\times F_{D}^{(3)}\left(1,1,1-m_{s d}, m_{s d} ; \frac{5}{2} ; \frac{1}{2}, \frac{G_{1, M Q A M}}{L_{1, M Q A M}}, \frac{G_{2, M Q A M}}{L_{2, M Q A M}}\right)
\end{array}
$$

where $L_{1, M Q A M}=1+4 b_{s d} \bar{\gamma}_{s d} g_{M Q A M}$ and $L_{2, M Q A M}=2 b_{s d} m_{s d}+4 b_{s d} \bar{\gamma}_{s d} g_{M Q A M}\left(2 b_{s d} m_{s d}+\Omega_{s d}\right)$. So, the average SEP of the direct link, $P_{s d, M Q A M}(E)$ is finally given by (22).

\section{B. Average SEP of SDF Hybrid/Integrated Satellite-Terrestrial Cooperative Network}

In this subsection, we evaluate the closed-form SEP of the SDF hybrid/integrated satellite-terrestrial cooperative network. The MGF of the total instantaneous received SNR at the MRC output is first derived. Then, we use this MGF to evaluate the average SEP. 


$$
\begin{aligned}
& P_{s d, M P S K}(E)=\frac{\left(2 b_{s d} m_{s d}\right)^{m_{s d}} G_{1, M P S K}^{m_{s d}-1}}{4 G_{2, M P S K}^{m_{s d}}} F_{1}\left(\frac{1}{2}, 1-m_{s d}, m_{s d} ; 2 ; \frac{1}{G_{1, M P S K}}, \frac{2 b_{s d} m_{s d}}{G_{2, M P S K}}\right) \\
& +\frac{\sqrt{\omega}\left(2 b_{s d} m_{s d}\right)^{m_{s d}} G_{1, M P S K}^{m_{s d}-1}}{\pi G_{2, M P S K}^{m_{s d}}} F_{D}^{(3)}\left(\frac{1}{2},-\frac{1}{2}, 1-m_{s d}, m_{s d} ; \frac{3}{2} ; \omega, \frac{\omega}{G_{1, M P S K}}, \frac{2 b_{s d} m_{s d} \omega}{G_{2, M P S K}}\right) . \\
& P_{s d, M Q A M}(E)=\frac{q\left(2 b_{s d} m_{s d}\right)^{m_{s d}} G_{1, M Q A M}^{m_{s d}-1}}{G_{2, M Q A M}^{m_{s d}}} F_{1}\left(\frac{1}{2}, 1-m_{s d}, m_{s d} ; 2 ; \frac{1}{G_{1, M Q A M}}, \frac{2 b_{s d} m_{s d}}{G_{2, M Q A M}}\right) \\
& -\frac{2 q^{2}\left(2 b_{s d} m_{s d}\right)^{m_{s d}} L_{1, M Q A M}^{m_{s d}-1}}{3 \pi L_{2, M Q A M}^{m_{s d}}} F_{D}^{(3)}\left(1,1,1-m_{s d}, m_{s d} ; \frac{5}{2} ; \frac{1}{2}, \frac{G_{1, M Q A M}}{L_{1, M Q A M}}, \frac{G_{2, M Q A M}}{L_{2, M Q A M}}\right) .
\end{aligned}
$$

1) MGF of the total received SNR: The instantaneous received SNR at the MRC combiner output is given by

$$
\gamma_{M R C}^{S D F}=\gamma_{s d}+\sum_{i \in C} \gamma_{r_{i} d}
$$

where $C$ is the decoding set with the cardinality $|C| \leq L$. Actually, it will be difficult to find the MGF of $\gamma_{M R C}^{S D F}$ given in (23) because the decoding set $C$ is unknown. To treat this problem we invoke the technique described in [27], where the system in Fig. 1 can be considered as a communication system consisting of $L+1$ effective paths between the satellite and the destination. Let path number 0 be the $s$ to $d$ direct link and path $i$ be the $s \rightarrow r_{i} \rightarrow d$ relayed link where $i=1, \ldots, L$. Let $\chi_{i}$ be the instantaneous received SNR of the relayed link $i$ at the destination which takes into account both the $s$ to $r_{i}$ link and the $r_{i}$ to $d$ link. Therefore, the PDF of $\chi_{i}$ can be obtained as

$$
\begin{aligned}
f_{\chi_{i}}(y) & =f_{\chi_{i} \mid r_{i} \text { Decodes Incorrectly }}(y) \operatorname{Pr}\left[r_{i} \text { Decodes Incorrectly }\right] \\
& +f_{\chi_{i} \mid r_{i} \text { Decodes Correctly }}(y) \operatorname{Pr}\left[r_{i} \text { Decodes Correctly }\right]
\end{aligned}
$$

The conditional PDF $f_{\chi_{i} \mid r_{i}}$ Decodes Incorrectly $(y)$ is given by

$$
f_{\chi_{i} \mid r_{i} \text { Decodes Incorrectly }}(y)=\delta(y)
$$

where $\delta(y)$ is the Dirac Delta function. And the conditional PDF $f_{\chi_{i} \mid r_{i} \text { Decodes Correctly }}(y)$ is given by

$$
\begin{array}{r}
f_{\chi_{i} \mid r_{i} \text { Decodes Correctly }}(y)=\frac{1}{\bar{\gamma}_{r_{i} d}} f_{h_{r_{i} d}^{2}}\left(\frac{y}{\bar{\gamma}_{r_{i} d}}\right) \\
=\frac{1}{2 b_{r_{i} d} \bar{\gamma}_{r_{i} d}} \exp \left(-\frac{y}{2 b_{r_{i} d} \bar{\gamma}_{r_{i} d}}\right)
\end{array}
$$

where $2 b_{r_{i} d}$ represents the average channel power gain of the Rayleigh fading and $\bar{\gamma}_{r_{i} d}=E_{r_{i}} / N_{0}$ is the average transmitted SNR per symbol of the relay $r_{i}$ to destination link. So, equation (23) can be rewritten as

$$
\gamma_{M R C}^{S D F}=\gamma_{s d}+\sum_{i=1}^{L} \chi_{i}
$$

The probability that the relay $r_{i}$ decodes incorrectly is the average SEP, $P_{s r_{i}}$, of the satellite-relay $r_{i}$ link. And the probability that the relay $r_{i}$ decodes correctly is $\left(1-P_{s r_{i}}\right)$. This $P_{s r_{i}}$ can be calculated by using the same approach as in (9) for the MPSK modulation scheme and as in (17) for the MQAM modulation scheme. The expressions of $P_{s r_{i}}$ are given by equations (28) and (29) for MPSK and MQAM modulation schemes respectively where

$$
\begin{aligned}
U_{1, M P S K} & =1+2 b_{s r_{i}} \bar{\gamma}_{s r_{i}} g_{M P S K}, \\
U_{2, M P S K} & =2 b_{s r_{i}} m_{s r_{i}} \\
& +2 b_{s r_{i}} \bar{\gamma}_{s r_{i}} g_{M P S K}\left(2 b_{s r_{i}} m_{s r_{i}}+\Omega_{s r_{i}}\right), \\
U_{1, M Q A M} & =1+2 b_{s r_{i}} \bar{\gamma}_{s r_{i}} g_{M Q A M}, \\
U_{2, M Q A M} & =2 b_{s r_{i}} m_{s r_{i}} \\
& +2 b_{s r_{i}} \bar{\gamma}_{s r_{i}} g_{M Q A M}\left(2 b_{s r_{i}} m_{s r_{i}}+\Omega_{s r_{i}}\right), \\
V_{1, M Q A M} & =1+4 b_{s r_{i}} \bar{\gamma}_{s r_{i}} g_{M Q A M}, \\
V_{2, M Q A M} & =2 b_{s r_{i}} m_{s r_{i}} \\
& +4 b_{s r_{i}} \bar{\gamma}_{s r_{i}} g_{M Q A M}\left(2 b_{s r_{i}} m_{s r_{i}}+\Omega_{s r_{i}}\right) .
\end{aligned}
$$

Therefore, equation (24) is given as

$$
f_{\chi_{i}}(y)=P_{s r_{i}} \delta(y)+\frac{\left(1-P_{s r_{i}}\right)}{2 b_{r_{i} d} \bar{\gamma}_{r_{i} d}} \exp \left(-\frac{y}{2 b_{r_{i} d} \bar{\gamma}_{r_{i} d}}\right) \text {. }
$$

The MGF of $\chi_{i}$ is expressed as

$$
\phi_{\chi_{i}}(s)=P_{s r_{i}}+\left(1-P_{s r_{i}}\right)\left(1+2 b_{r_{i} d} \bar{\gamma}_{r_{i} d} s\right)^{-1} \text {. }
$$

So the MGF of $\gamma_{M R C}^{S D F}$ is finally obtained as

$$
\phi_{\gamma_{M R C}^{S D F}}(s)=\phi_{\gamma_{s d}}(s) \prod_{i=1}^{L} \phi_{\chi_{i}}(s) \text {. }
$$

2) M-ary Phase-Shift Keying (MPSK): The average SEP of the SDF hybrid/integrated satellite-terrestrial cooperative systems for coherent MPSK signals is given by [25]

$$
P_{M R C, M P S K}^{S D F}(E)=\frac{1}{\pi} \int_{0}^{\pi-\frac{\pi}{M}} \phi_{\gamma_{M R C}^{S D F}}\left(\frac{g_{M P S K}}{\sin ^{2} \theta}\right) d \theta
$$

where $g_{M P S K}=\sin ^{2}(\pi / M)$. By using the same manipulation as in (9), we can get $P_{s, M P S K}^{S D F}(E)$ as shown in equation (34) where $H_{i, M P S K}=1+P_{s r_{i}, M P S K} 2 b_{r_{i} d} \bar{\gamma}_{r_{i} d} g_{M P S K}$ and $K_{i, M P S K}=1+2 b_{r_{i} d} \bar{\gamma}_{r_{i} d} g_{M P S K}$.

In the case of independent and identically distributed (i.i.d) fading channels, all relays are experiencing the same fading environment, i.e., $m_{s r_{i}}=m_{s r}, b_{s r_{i}}=b_{s r}, \Omega_{s r_{i}}=\Omega_{s r}$ and $b_{r_{i} d}=b_{r d}$ for all $i \in L$. And we assume that $\bar{\gamma}_{r_{i} d}=$ $\bar{\gamma}_{r d}=\bar{\gamma}$ for all $i \in L$. So, $H_{i, M P S K}=H_{M P S K}$ and $K_{i, M P S K}=K_{M P S K}$ for all $i \in L$. By using the properties 


$$
\begin{aligned}
& P_{s r_{i}, M P S K}(E)=\frac{\left(2 b_{s r_{i}} m_{s r_{i}}\right)^{m_{s r_{i}}} U_{1, M P S K}^{m_{s r_{i}}-1}}{4 U_{2, M P S K}^{m_{s r_{i}}}} F_{1}\left(\frac{1}{2}, 1-m_{s r_{i}}, m_{s r_{i}} ; 2 ; \frac{1}{U_{1, M P S K}}, \frac{2 b_{s r_{i}} m_{s r_{i}}}{U_{2, M P S K}}\right) \\
& +\frac{\sqrt{\omega}\left(2 b_{s r_{i}} m_{s r_{i}}\right)^{m_{s r_{i}}} U_{1, M P S K}^{m_{s r_{i}}-1}}{\pi U_{2, M P S K}^{m_{s r_{i}}}} F_{D}^{(3)}\left(\frac{1}{2},-\frac{1}{2}, 1-m_{s r_{i}}, m_{s r_{i}} ; \frac{3}{2} ; \omega, \frac{\omega}{U_{1, M P S K}}, \frac{2 b_{s r_{i}} m_{s r_{i}} \omega}{U_{2, M P S K}}\right) \\
& P_{s r_{i}, M Q A M}(E)=\frac{q\left(2 b_{s r_{i}} m_{s r_{i}}\right)^{m_{s r_{i}}} U_{1, M Q A M}^{m_{s r_{i}}-1}}{U_{2, M Q A M}^{m_{s r_{i}}}} F_{1}\left(\frac{1}{2}, 1-m_{s r_{i}}, m_{s r_{i}} ; 2 ; \frac{1}{U_{1, M Q A M}}, \frac{2 b_{s r_{i}} m_{s r_{i}}}{U_{2, M Q A M}}\right) \\
& -\frac{2 q^{2}\left(2 b_{s r_{i}} m_{s r_{i}}\right)^{m_{s r_{i}}} V_{1, M Q A M}^{m_{s r_{i}}-1}}{3 \pi V_{2, M Q A M}^{m_{s r_{i}}}} F_{D}^{(3)}\left(1,1,1-m_{s r_{i}}, m_{s r_{i}} ; \frac{5}{2} ; \frac{1}{2}, \frac{U_{1, M Q A M}}{V_{1, M Q A M}}, \frac{U_{2, M Q A M}}{V_{2, M Q A M}}\right) \\
& P_{M R C, M P S K}^{S D F}(E)=\frac{\left(2 b_{s d} m_{s d}\right)^{m_{s d}} G_{1, M P S K}^{m_{s d}-1}}{4 G_{2, M P S K}^{m_{s d}}} \prod_{i=1}^{L}\left(\frac{H_{i, M P S K}}{K_{i, M P S K}}\right) F_{D}^{(2 L+2)}\left(\frac{1}{2}, 1-m_{s d}, m_{s d},-1,1, \ldots,-1,1\right. \\
& \left.; 2 ; \frac{1}{G_{1, M P S K}}, \frac{2 b_{s d} m_{s d}}{G_{2, M P S K}}, \frac{1}{H_{1, M P S K}}, \frac{1}{K_{1, M P S K}}, \ldots, \frac{1}{H_{L, M P S K}}, \frac{1}{K_{L, M P S K}}\right) \\
& +\frac{\sqrt{\omega}\left(2 b_{s d} m_{s d}\right)^{m_{s d}} G_{1, M P S K}^{m_{s d}-1}}{\pi G_{2, M P S K}^{m_{s d}}} \prod_{i=1}^{L}\left(\frac{H_{i, M P S K}}{K_{i, M P S K}}\right) F_{D}^{(2 L+3)}\left(\frac{1}{2},-\frac{1}{2}, 1-m_{s d}, m_{s d},-1,1, \ldots\right. \\
& \left.,-1,1 ; \frac{3}{2} ; \omega, \frac{\omega}{G_{1, M P S K}}, \frac{2 b_{s d} m_{s d} \omega}{G_{2, M P S K}}, \frac{\omega}{H_{1, M P S K}}, \frac{\omega}{K_{1, M P S K}}, \ldots, \frac{\omega}{H_{L, M P S K}}, \frac{\omega}{K_{L, M P S K}}\right)
\end{aligned}
$$

of the Lauricella function as shown below

$$
\begin{array}{r}
F_{D}^{(2 L+N)}\left(a, b_{1}, \ldots, b_{N}, d, e, \ldots, d, e ; c\right. \\
\left.; x_{1}, \ldots, x_{N}, y, z, \ldots, y, z\right) \\
=F_{D}^{(N+2)}\left(a, b_{1}, \ldots, b_{N}, L d, L e ; c ; x_{1}, \ldots, x_{N}, y, z\right) \\
\text { for }|y|<1 \text { and }|z|<1
\end{array}
$$

the average SEP, $P_{M R C, M P S K}^{S D F}(E)$ can be simplified as in equation (36) which indicates that a full diversity order of $L+1$ is obtained when the number of participating relays is $L$ (see appendix A).

3) M-ary Quadrature Amplitude Modulation (MQAM):

The average SEP of the SDF hybrid/integrated satelliteterrestrial cooperative systems for coherent MQAM signals is given by [25]

$$
\begin{aligned}
P_{M R C, M Q A M}^{S D F}(E) & =\frac{4 q}{\pi} \int_{0}^{\frac{\pi}{2}} \phi_{\gamma_{M R C}^{S D F}}\left(\frac{g_{M Q A M}}{\sin ^{2} \theta}\right) d \theta \\
& -\frac{4 q^{2}}{\pi} \int_{0}^{\frac{\pi}{4}} \phi_{\gamma_{M R C}^{S D F}}\left(\frac{g_{M Q A M}}{\sin ^{2} \theta}\right) d \theta
\end{aligned}
$$

where $g_{M Q A M}=3 / 2(M-1)$ and $q=(1-1 / \sqrt{M})$. $P_{M R C, M Q A M}^{S D F}$ can be calculated by using the same approach as in (17). Hence, the average SEP $P_{M R C, M Q A M}^{S D F}$ is given by equation (38) where

$$
\begin{aligned}
H_{i, M Q A M} & =1+P_{s r_{i}, M Q A M} 2 b_{r_{i} d} \bar{\gamma}_{r_{i} d} g_{M Q A M}, \\
K_{i, M Q A M} & =1+2 b_{r_{i} d} \bar{\gamma}_{r_{i} d} g_{M Q A M}, \\
W_{i, M Q A M} & =1+P_{s r_{i}, M Q A M} 4 b_{r_{i} d} \bar{\gamma}_{r_{i} d} g_{M Q A M}, \\
Z_{i, M Q A M} & =1+4 b_{r_{i} d} \bar{\gamma}_{r_{i} d} g_{M Q A M} .
\end{aligned}
$$

In the case of i.i.d fading channels, all relays are experiencing the same fading environment, i.e., $m_{s r_{i}}=m_{s r}$, $b_{s r_{i}}=b_{s r}, \Omega_{s r_{i}}=\Omega_{s r}$ and $b_{r_{i} d}=b_{r d}$ for all $i \in L$. And we assume that $\bar{\gamma}_{r_{i} d}=\bar{\gamma}_{r d}=\bar{\gamma}$ for all $i \in L$. So, $H_{i, M Q A M}=$ $H_{M Q A M}, K_{i, M Q A M}=K_{M Q A M}, W_{i, M Q A M}=W_{M Q A M}$ and $Z_{i, M Q A M}=Z_{M Q A M}$ for all $i \in L$. By using the properties of the Lauricella function as shown in (35), the average SEP, $P_{s, M Q A M}^{S D F}(E)$ can be simplified as in equation (39) which again indicates that a full diversity order of $L+1$ is obtained when the number of participating relays is $L$ (see appendix B).

\section{Simulation Results}

In this section, simulations and numerical results of the average SEP of a hybrid/integrated satellite-terrestrial cooperative network are evaluated. The SEP curves are plotted versus the average transmitted SNR normalized to the long term path loss for different number of relays $L=0,1,2,3,4,5$ ( $L=0$, corresponds to the direct transmission only). In order to show the diversity order, we assume that the average transmitted SNR per symbol of the satellite-destination link is equal to the one of the relay-destination links $\left(E_{s} / N_{0}=E_{r_{i}} / N_{0}\right)$. The numerical values for the LMS channel are shown in Table I and the relay-destination links are Rayleigh fading channel with the average channel power gain equal to unity. We can observe from the figures that our analytical results show excellent agreement with the simulation results for both MPSK (Figs. 2 and 3) and MQAM (Fig. 4) modulation schemes and for all cases of fading channels. This confirms the accuracy of our performance analysis. We also compare SEPs of our proposed SDF system with the regular FDF system as shown in Figs 2, 3 and 4. These three figures shows that our proposed SDF system outperforms the regular FDF system in terms of SEP. 


$$
\begin{aligned}
& P_{M R C, M P S K}^{S D F}(E)=\frac{\left(2 b_{s d} m_{s d}\right)^{m_{s d}} G_{1, M P S K}^{m_{s d}-1}}{4 G_{2, M P S K}^{m_{s d}}}\left(\frac{H_{M P S K}}{K_{M P S K}}\right)^{L} \\
& \times F_{D}^{(4)}\left(\frac{1}{2}, 1-m_{s d}, m_{s d},-L, L ; 2 ; \frac{1}{G_{1, M P S K}}, \frac{2 b_{s d} m_{s d}}{G_{2, M P S K}}, \frac{1}{H_{M P S K}}, \frac{1}{K_{M P S K}}\right) \\
& +\frac{\sqrt{\omega}\left(2 b_{s d} m_{s d}\right)^{m_{s d}} G_{1, M P S K}^{m_{s d}-1}}{\pi G_{2, M P S K}^{m_{s d}}}\left(\frac{H_{M P S K}}{K_{M P S K}}\right)^{L} \\
& \times F_{D}^{(5)}\left(\frac{1}{2},-\frac{1}{2}, 1-m_{s d}, m_{s d},-L, L ; \frac{3}{2} ; \omega, \frac{\omega}{G_{1, M P S K}}, \frac{2 b_{s d} m_{s d} \omega}{G_{2, M P S K}}, \frac{\omega}{H_{M P S K}}, \frac{\omega}{K_{M P S K}}\right) . \\
& P_{M R C, M Q A M}^{S D F}(E)=\frac{q\left(2 b_{s d} m_{s d}\right)^{m_{s d}} G_{1, M Q A M}^{m_{s d}-1}}{G_{2, M Q A M}^{m_{s d}}} \prod_{i=1}^{L}\left(\frac{H_{i, M Q A M}}{K_{i, M Q A M}}\right) F_{D}^{(2 L+2)}\left(\frac{1}{2}, 1-m_{s d}, m_{s d},-1,1, \ldots,-1,1\right. \\
& \left.; 2 ; \frac{1}{G_{1, M Q A M}}, \frac{2 b_{s d} m_{s d}}{G_{2, M Q A M}}, \frac{1}{H_{1, M Q A M}}, \frac{1}{K_{1, M Q A M}}, \ldots, \frac{1}{H_{L, M Q A M}}, \frac{1}{K_{L, M Q A M}}\right) \\
& -\frac{2 q^{2}\left(2 b_{s d} m_{s d}\right)^{m_{s d}} L_{1, M Q A M}^{m_{s d}-1}}{3 \pi L_{2, M Q A M}^{m_{s d}}} \prod_{i=1}^{L}\left(\frac{W_{i, M Q A M}}{Z_{i, M Q A M}}\right) F_{D}^{(2 L+3)}\left(1,1,1-m_{s d}, m_{s d},-1,1, \ldots\right. \\
& \left.,-1,1 ; \frac{5}{2} ; \frac{1}{2}, \frac{G_{1, M Q A M}}{L_{1, M Q A M}}, \frac{G_{2, M Q A M}}{L_{2, M Q A M}}, \frac{H_{1, M Q A M}}{W_{1, M Q A M}}, \frac{K_{1, M Q A M}}{Z_{1, M Q A M}}, \ldots, \frac{H_{L, M Q A M}}{W_{L, M Q A M}}, \frac{K_{L, M Q A M}}{Z_{L, M Q A M}}\right) . \\
& P_{M R C, M Q A M}^{S D F}(E)=\frac{q\left(2 b_{s d} m_{s d}\right)^{m_{s d}} G_{1, M Q A M}^{m_{s d}-1}}{G_{2, M Q A M}^{m_{s d}}}\left(\frac{H_{M Q A M}}{K_{M Q A M}}\right)^{L} \\
& \times F_{D}^{(4)}\left(\frac{1}{2}, 1-m_{s d}, m_{s d},-L, L ; 2 ; \frac{1}{G_{1, M Q A M}}, \frac{2 b_{s d} m_{s d}}{G_{2, M Q A M}}, \frac{1}{H_{M Q A M}}, \frac{1}{K_{M Q A M}}\right) \\
& -\frac{2 q^{2}\left(2 b_{s d} m_{s d}\right)^{m_{s d}} L_{1, M Q A M}^{m_{s d}-1}}{3 \pi L_{2, M Q A M}^{m_{s d}}}\left(\frac{W_{M Q A M}}{Z_{M Q A M}}\right)^{L} \\
& \times F_{D}^{(5)}\left(1,1,1-m_{s d}, m_{s d},-L, L ; \frac{5}{2} ; \frac{1}{2}, \frac{G_{1, M Q A M}}{L_{1, M Q A M}}, \frac{G_{2, M Q A M}}{L_{2, M Q A M}}, \frac{H_{M Q A M}}{W_{M Q A M}}, \frac{K_{M Q A M}}{Z_{M Q A M}}\right) .
\end{aligned}
$$

TABLE I

LMS CHANNEL PARAMETERS [22]

\begin{tabular}{c|c|c|c|}
\multirow{2}{*}{} & \multicolumn{3}{|c|}{ Data set } \\
\cline { 2 - 4 } & $b_{s x}$ & $m_{s x}$ & $\Omega_{s x}$ \\
\hline Frequent heavy shadowing & 0.063 & 0.739 & $8.97 \times 10^{-4}$ \\
\hline Average shadowing & 0.126 & 10.1 & 0.835 \\
\hline Infrequent light shadowing & 0.158 & 19.4 & 1.29 \\
\hline
\end{tabular}

Fig. 2 shows the average SEP of QPSK hybrid/integrated satellite-terrestrial cooperative systems when both direct link and satellite-relay links experience the frequent heavy shadowed fading environment. Moreover, the diversity gain increases as a function of the number of participating relays. For example, we can achieve the diversity gain around $7 \mathrm{~dB}$ at the SEP of $10^{-1}$ when only one relay is used. In addition, this diversity gain does increase to approximately $12 \mathrm{~dB}$ when $L=3$ and $14.5 \mathrm{~dB}$ when $L=5$.

Fig. 3 plots the average SEP of 8PSK hybrid/integrated satellite-terrestrial cooperative systems when the direct link is under the frequent heavy shadowed fading condition and the satellite-relay links are under the average shadowed fading condition. We can observe from the figure that the 3-relay system gains $5.8 \mathrm{~dB}$ for $\mathrm{SEP}=10^{-1}$ over the 1-relay system

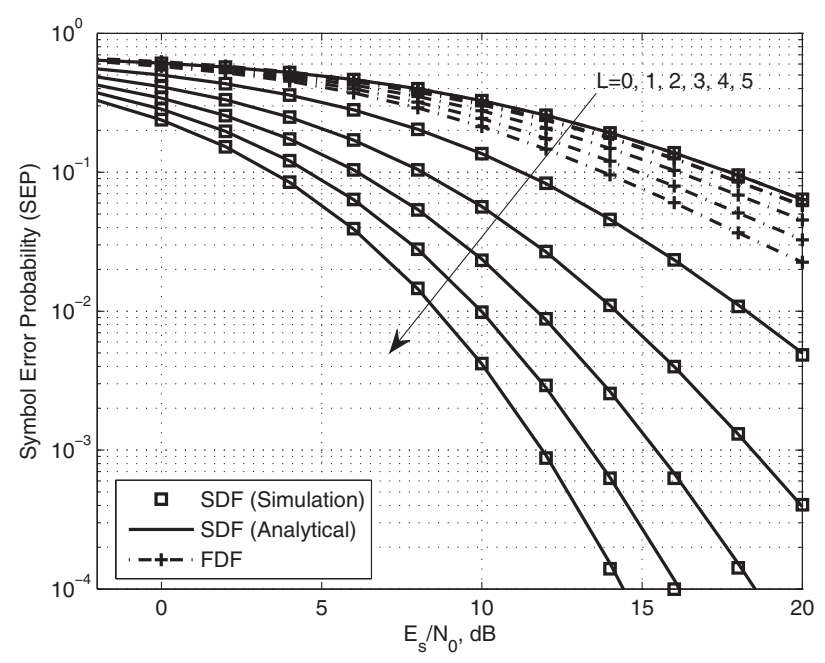

Fig. 2. The average SEP of QPSK versus the average transmitted SNR, $E_{s} / N_{0}$, when both direct and satellite-relay links experience the frequent heavy shadowing.

while the 5-relay system gains $7.8 \mathrm{~dB}$ over the 1-relay system for the same SEP. 


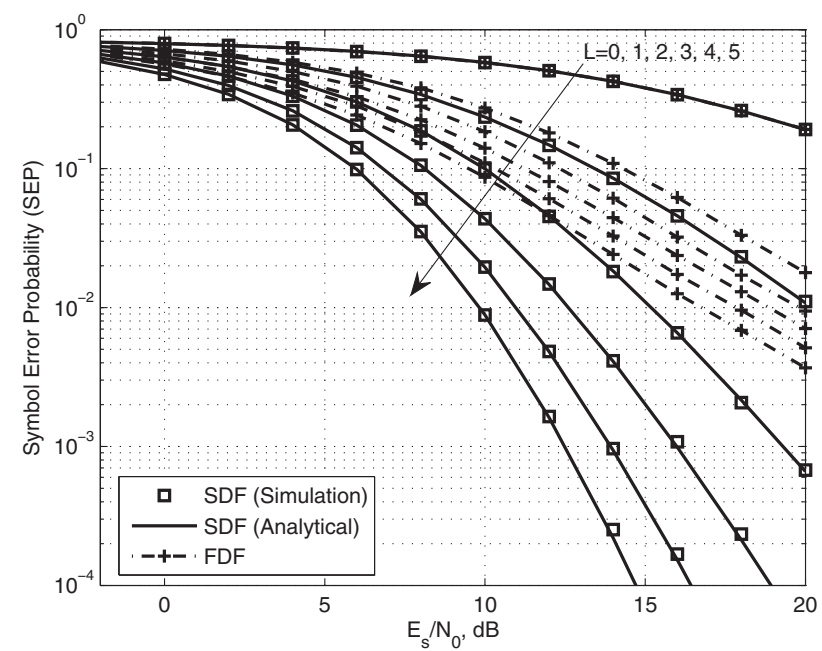

Fig. 3. The average SEP of 8PSK versus the average transmitted SNR, $E_{s} / N_{0}$, when the direct link experiences the frequent heavy shadowing and satellite-relay links experience the average shadowing.

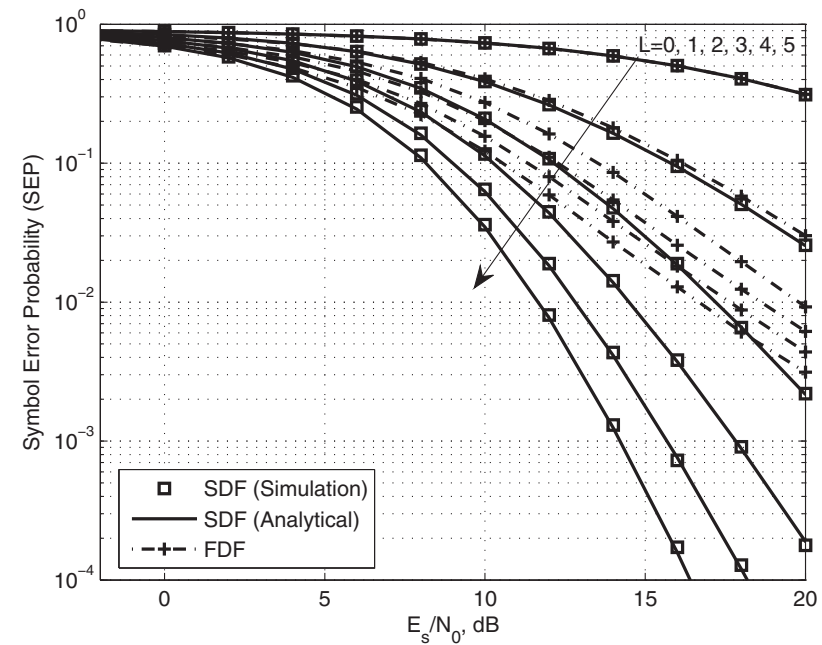

Fig. 4. The average SEP of 16QAM versus the average transmitted SNR, $E_{s} / N_{0}$, when the direct link experiences the frequent heavy shadowing and satellite-relay links experience the infrequent light shadowing.

Furthermore, Fig. 4 illustrates the average SEP of 16QAM hybrid/integrated satellite-terrestrial cooperative systems when the direct link experiences the frequent heavy shadowing and the satellite-relay links are under the infrequent light shadowed fading condition. We can see in the Fig. 4 that the 3-relay system gains $6 \mathrm{~dB}$ for $\mathrm{SEP}=10^{-1}$ over the 1-relay system and the 5-relay system gains $8 \mathrm{~dB}$ over the 1-relay system for the same SEP.

Fig. 5 plots the theoretical SEP curves of 16PSK-SDF and 16QAM-SDF hybrid/integrated satellite-terrestrial cooperative systems while Fig. 6 plots the theoretical SEP curves of 64PSK-SDF and 64QAM-SDF hybrid/integrated satelliteterrestrial cooperative systems when the direct link experiences the frequent heavy shadowing and satellite-relay links experience the infrequent light shadowing. Although both modulation schemes provide the same diversity order, the

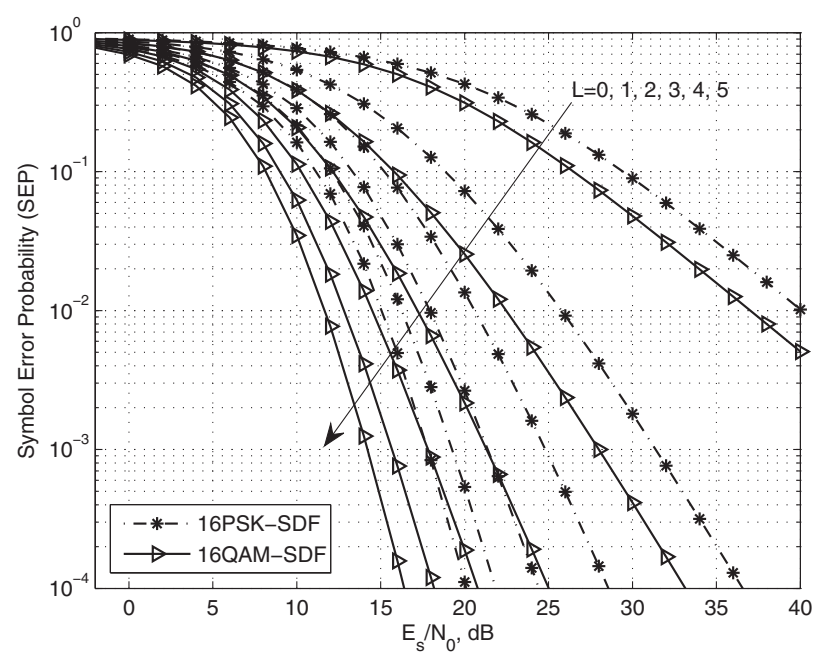

Fig. 5. Theoretical SEP curves of 16PSK and 16QAM versus the average transmitted SNR, $E_{s} / N_{0}$, when the direct link experiences the frequent heavy shadowing and satellite-relay links experience the infrequent light shadowing.

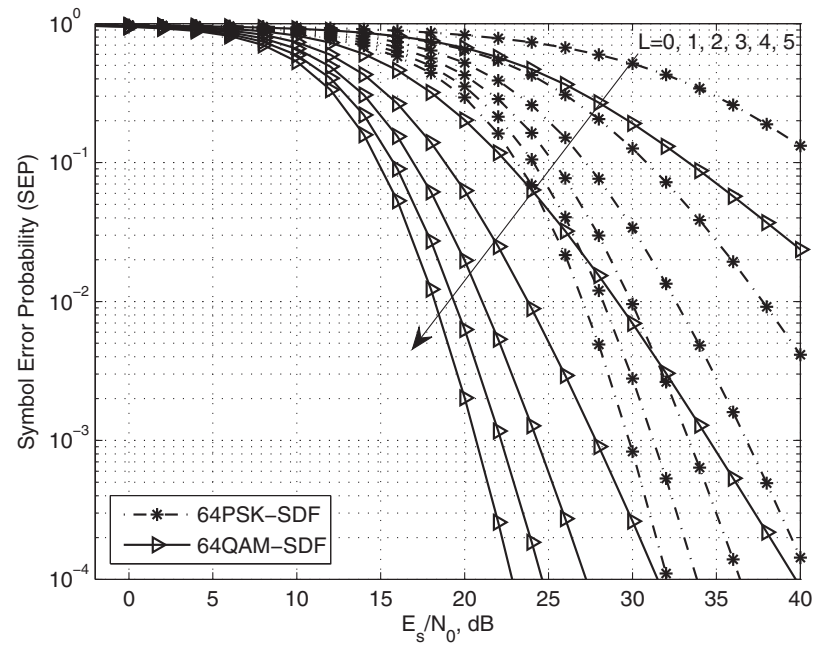

Fig. 6. Theoretical SEP curves of 64PSK and 64QAM versus the average transmitted SNR, $E_{s} / N_{0}$, when the direct link experiences the frequent heavy shadowing and satellite-relay links experience the infrequent light shadowing.

MQAM-SDF outperforms the MPSK-SDF in terms of SEP for the same number of constellation points $M$ in the highSNR regime. From Fig. 5, we can see that the 16QAM-SDF provides $3 \mathrm{~dB}$ of diversity gain over the 16PSK-SDF for $\mathrm{SEP}=10^{-1}$. From Fig. 6, we can also notice that 64QAMSDF provides $8 \mathrm{~dB}$ of diversity gain over the 64PSK-SDF for SEP $=10^{-1}$. These results are consistent with known results on the power efficiency of these modulation schemes.

\section{CONClusion}

In this paper, we studied the performance in terms of SEP of a hybrid/integrated satellite-terrestrial cooperative network. The SDF transmission scheme has been implemented. In the first phase, the satellite broadcasts its signal to all relay nodes and the destination node. In the second phase, only relays that can decode the satellite message correctly are 
allowed to forward the satellite message to the destination node. The exact closed-form expressions for the average SEP of the general MPSK and MQAM hybrid/integrated satellite-terrestrial cooperative systems over independent but not necessarily identically distributed fading channels have been derived. The results have shown that a full diversity order of $L+1$ can be obtained when the total number of relays is equal to $L$. Moreover, it can be seen from the SEP curves that our analytical expressions show excellent agreement with the simulation results. The obtained SEP expressions will provide valuable insight into the design of the hybrid/integrated satellite-terrestrial cooperative systems especially in the emergency communication systems.

\section{APPENDIX A}

ASYMPTOTIC DIVERSITY ORDER OF $P \underset{M R C, M P S K}{S D F}(E)$

In order to show the diversity order, we assume that $\bar{\gamma}_{s d}=\bar{\gamma}_{s r_{i}}=\bar{\gamma}_{r_{i} d}=\bar{\gamma}$.

The asymptotic diversity order $D_{g, M P S K}$ of $P_{M R C, M P S K}^{S D F}(E)$ is given in [28] as

$$
D_{g, M P S K}=-\lim _{\bar{\gamma} \rightarrow+\infty} \frac{\log P_{M R C, M P S K}^{S D F}(\bar{\gamma})}{\log (\bar{\gamma})} .
$$

By using the equation (36), the asymptotic diversity order $D_{g, M P S K}$ can be rewritten as

$$
D_{g, M P S K}=-\lim _{\bar{\gamma} \rightarrow+\infty} \frac{1}{\log (\bar{\gamma})} \times \log \left(A_{1} \times A_{2} \times A_{3} \times A_{4}\right)
$$

$$
\text { where } A_{1}=\left(2 b_{s d} m_{s d}\right)^{m_{s d}}, A_{2}=\frac{G_{1, M P S K}^{m_{s d}-1}}{G_{2, M P S K}^{m_{s d}}} \text {, }
$$

$$
\begin{aligned}
A_{3} & =\left(\frac{H_{M P S K}}{K_{M P S K}}\right)^{L}, \\
A_{4} & =\frac{1}{4} F_{D}^{(4)}\left(\frac{1}{2}, 1-m_{s d}, m_{s d},-L, L ; 2 ;\right. \\
& \left.\frac{1}{G_{1, M P S K}}, \frac{2 b_{s d} m_{s d}}{G_{2, M P S K}}, \frac{1}{H_{M P S K}}, \frac{1}{K_{M P S K}}\right) \\
& +\frac{\sqrt{\omega}}{\pi} F_{D}^{(5)}\left(\frac{1}{2},-\frac{1}{2}, 1-m_{s d}, m_{s d},-L, L ; \frac{3}{2} ;\right. \\
\omega, & \left.\frac{\omega}{G_{1, M P S K}}, \frac{2 b_{s d} m_{s d} \omega}{G_{2, M P S K}}, \frac{\omega}{H_{M P S K}}, \frac{\omega}{K_{M P S K}}\right)
\end{aligned}
$$

At the high SNR regime, we obtain

$$
\begin{array}{r}
A_{2} \approx \frac{1}{\bar{\gamma}}, P_{s r, M P S K} \approx c_{1} \times \frac{1}{\bar{\gamma}} \Rightarrow A_{3} \approx\left(\frac{c_{2}}{\bar{\gamma}}\right)^{L}, \\
A_{4} \approx \frac{1}{4} F_{D}^{(4)}\left(\frac{1}{2}, 1-m_{s d}, m_{s d},-L, L ; 2 ; 0,0, c_{3}, 0\right) \\
+\frac{\sqrt{\omega}}{\pi} F_{D}^{(5)}\left(\frac{1}{2},-\frac{1}{2}, 1-m_{s d}, m_{s d},-L, L ; \frac{3}{2} ; \omega, 0,0, c_{4}, 0\right)
\end{array}
$$

where the symbol $\approx$ denotes the approximation for the high SNR regime and $c_{i}(i=1,2,3,4)$ is a constant value. We observe that in the high SNR regime, $A_{4}$ converges to a constant value. So, the asymptotic diversity $D_{g, M P S K}$ is finally given as

$$
\begin{array}{r}
D_{g, M P S K}=-\lim _{\bar{\gamma} \rightarrow+\infty} \frac{1}{\log (\bar{\gamma})} \\
\times\left[\log \left(A_{1}\right)+\log \left(\frac{1}{\bar{\gamma}}\right)+\log \left(\frac{c_{2}}{\bar{\gamma}}\right)^{L}+\log \left(A_{4}\right)\right]=L+1 .
\end{array}
$$

\section{APPENDIX B}

ASYMPTOTIC DIVERSITY ORDER OF P PRC,MQAM $(E)$

In order to show the diversity order, we assume that

$\bar{\gamma}_{s d}=\bar{\gamma}_{s r_{i}}=\bar{\gamma}_{r_{i} d}=\bar{\gamma}$.

The asymptotic diversity order $D_{g, M Q A M}$ of $P_{M R C, M Q A M}^{S D F}(E)$ is given in [28] as

$$
D_{g, M Q A M}=-\lim _{\bar{\gamma} \rightarrow+\infty} \frac{\log P_{M R C, M Q A M}^{S D F}(\bar{\gamma})}{\log (\bar{\gamma})} .
$$

By using the equation (39), the asymptotic diversity order $D_{g, M Q A M}$ can be rewritten as

$$
\begin{array}{r}
D_{g, M Q A M}=-\lim _{\bar{\gamma} \rightarrow+\infty} \frac{1}{\log (\bar{\gamma})} \\
\times \log \left(B_{1} \times B_{2} \times B_{3} \times B_{4}-C_{1} \times C_{2} \times C_{3} \times C_{4}\right)
\end{array}
$$

where $B_{1}=q\left(2 b_{s d} m_{s d}\right)^{m_{s d}}, B_{2}=\frac{G_{1, M Q A M}^{m_{s d}-1}}{G_{2, M Q A M}^{m_{s d}}}$,

$$
\begin{aligned}
B_{3} & =\left(\frac{H_{M Q A M}}{K_{M Q A M}}\right)^{L}, \\
B_{4} & =F_{D}^{(4)}\left(\frac{1}{2}, 1-m_{s d}, m_{s d},-L, L ; 2 ;\right. \\
& \left.\quad \frac{1}{G_{1, M Q A M}}, \frac{2 b_{s d} m_{s d}}{G_{2, M Q A M}}, \frac{1}{H_{M Q A M}}, \frac{1}{K_{M Q A M}}\right), \\
C_{1} & =\frac{2 q^{2}\left(2 b_{s d} m_{s d}\right)^{m_{s d}}}{3 \pi}, C_{2}=\frac{L_{1, M Q A M}^{m_{s d}-1}}{L_{2, M Q A M}^{m_{s d}}}, \\
C_{3} & =\left(\frac{W_{M Q A M}}{Z_{M Q A M}}\right)^{L}, \\
C_{4} & =F_{D}^{(5)}\left(1,1,1-m_{s d}, m_{s d},-L, L ; \frac{5}{2} ;\right. \\
& \left.\frac{1}{2}, \frac{G_{1, M Q A M}}{L_{1, M Q A M}}, \frac{G_{2, M Q A M}}{L_{2, M Q A M}}, \frac{H_{M Q A M}}{W_{M Q A M}}, \frac{K_{M Q A M}}{Z_{M Q A M}}\right) .
\end{aligned}
$$

At the high SNR regime, we have that

$$
\begin{array}{r}
B_{2} \approx \frac{1}{\bar{\gamma}}, P_{s r, M Q A M} \approx e_{1} \times \frac{1}{\bar{\gamma}} \Rightarrow B_{3} \approx\left(\frac{e_{2}}{\bar{\gamma}}\right)^{L}, \\
B_{4} \approx F_{D}^{(4)}\left(\frac{1}{2}, 1-m_{s d}, m_{s d},-L, L ; 2 ; 0,0, e_{3}, 0\right), \\
C_{2} \approx \frac{1}{\bar{\gamma}}, C_{3} \approx\left(\frac{e_{4}}{\bar{\gamma}}\right)^{L}, \\
C_{4} \approx F_{D}^{(5)}\left(1,1,1-m_{s d}, m_{s d},-L, L ; \frac{5}{2} ; \frac{1}{2}, \frac{1}{2}, \frac{1}{2}, e_{5}, \frac{1}{2}\right)
\end{array}
$$

where $e_{i}(i=1,2,3,4,5)$ is a constant value. We observe that in the high SNR regime, $B_{4}$ and $C_{4}$ converge to constant 
values. So, the asymptotic diversity $D_{g, M Q A M}$ is given as

$$
\begin{aligned}
D_{g, M Q A M}= & -\lim _{\bar{\gamma} \rightarrow+\infty} \frac{1}{\log (\bar{\gamma})} \times \log \left[B_{1} \times\left(\frac{1}{\bar{\gamma}}\right) \times\left(\frac{e_{2}}{\bar{\gamma}}\right)^{L}\right. \\
& \left.\times B_{4}-C_{1} \times\left(\frac{1}{\bar{\gamma}}\right) \times\left(\frac{e_{4}}{\bar{\gamma}}\right)^{L} \times C_{4}\right] \\
D_{g, M Q A M} & =-\lim _{\bar{\gamma} \rightarrow+\infty} \frac{1}{\log (\bar{\gamma})} \times\left[\log \left(\frac{1}{\bar{\gamma}}\right)^{(L+1)}\right. \\
& \left.+\log \left(B_{1} \times e_{2}^{L} \times B_{4}-C_{1} \times e_{4}^{L} \times C_{4}\right)\right] \\
& =L+1 .
\end{aligned}
$$

\section{REFERENCES}

[1] P. Chini, G. Giambene, and S. Kota, "A survey on mobile satellite systems," Int. J. Sat. Commun., vol. 28, pp. 29-57, 2009.

[2] G. G. Kota and S. Kim, "Satellite component of NGN: integrated and hybrid networks," Int. J. Sat. Commun., vol. 29, pp. 191-208, 2010.

[3] B. Evans, M. Werner, E. Lutz, M. Bousquet, G. Corazza, G. Maral, and R. Rumeau, "Integration of satellite and terrestrial systems in future multimedia communications," IEEE Wireless Commun., vol. 12, no. 5 , pp. 72-80, Oct. 2005.

[4] N. Chuberre, O. Courseille, P. Laine, L. Roullet, T. Quignon, and M. Tatard, "Hybrid satellite and terrestrial infrastructure for mobile broadcast services delivery: an outlook to the unlimited mobile TV system performance," Int. J. Sat. Commun., vol. 26, pp. 405-426, 2008.

[5] J. Laneman, D. Tse, and G. Wornell, "Cooperative diversity in wireless networks: efficient protocols and outage behavior," IEEE Trans. Inf. Theory, vol. 50, no. 12, pp. 3062-3080, Dec. 2004.

[6] J. Laneman and G. Wornell, "Distributed space-time-coded protocols for exploiting cooperative diversity in wireless networks," IEEE Trans. Inf. Theory, vol. 49, no. 10, pp. 2415-2425, Oct. 2003.

[7] G. Iapichino, C. Bonnet, O. del Rio Herrero, C. Baudoin, and I. Buret, "Advanced hybrid satellite and terrestrial system architecture for emergency mobile communications," in Proc. 2008 AIAA International Communications Satellite Systems Conference, pp. 1-8.

[8] E. Del Re, S. Morosi, S. Jayousi, and C. Sacchi, "Salice-satelliteassisted localization and communication systems for emergency services," in Proc. 2009 International Conference on Wireless Communication, Vehicular Technology, Information Theory and Aerospace Electronic Systems Technology, pp. 544-548.

[9] S. Morosi, S. Jayousi, and E. Del Re, "Cooperative delay diversity in hybrid satellite/terrestrial DVB-SH system," in Proc. 2010 IEEE International Conference on Communications, pp. 1-5.

[10] D.-S. Ahn, S. Kim, H. W. Kim, and D.-C. Park, "A cooperative transmit diversity scheme for mobile satellite broadcasting systems." Int. J. Sat. Commun., vol. 28, pp. 352-368, 2010.

[11] G. Cocco, C. Ibars, and O. del Rio Herrero, "Cooperative satellite to land mobile gap-filler-less interactive system architecture," in Proc. 2010 Advanced Satellite Multimedia Systems Conference and the 2010 Signal Processing for Space Communications Workshop, pp. 309-314.

[12] B. Paillassa, B. Escrig, R. Dhaou, M.-L. Boucheret, and C. Bes, "Improving satellite services with cooperative communications," Int. J. Sat. Commun., vol. 29, pp. 479-500, 2011.

[13] A. Iqbal and K. M. Ahmed, "A hybrid satellite-terrestrial cooperative network over non identically distributed fading channels," J. Commun., vol. 7, pp. 581-589, 2011.

[14] C. Loo, "A statistical model for a land mobile satellite link," IEEE Trans. Veh. Technol., vol. 34, no. 3, pp. 122-127, Aug. 1985.

[15] _ - "Digital transmission through a land mobile satellite channel," IEEE Trans. Commun., vol. 38, no. 5, pp. 693-697, May 1990.

[16] — " "Further results on the statistics of propagation data at Lband (1542 MHz) for mobile satellite communications," in 1991 IEEE Vehicular Technology Conf. - Spring, pp. 51-56.

[17] C. Loo and J. Butterworth, "Land mobile satellite channel measurement and modeling," Proc. IEEE, vol. 86, no. 7, pp. 1442-1463, Jul. 1998.

[18] E. Lutz, D. Cygan, M. Dippold, F. Dolainsky, and W. Papke, "The land mobile satellite communication channel-recording, statistics, and channel model," IEEE Trans. Veh. Technol., vol. 40, no. 2, pp. 375386, May 1991.

[19] F. Perez-Fontan, V. Hovinen, M. Schonhuber, R. Prieto-Cerdeira, J. Rivera-Castro, P. Valtr, J. Kyrolainen, and F. Teschl, "Characterisation of the satellite-to-indoor propagation channel," in Proc. 2008 Advanced Satellite Mobile Systems, pp. 105-110.
[20] F. Perez-Fontan, M. Vazquez-Castro, S. Buonomo, J. Poiares-Baptista, and B. Arbesser-Rastburg, "S-band LMS propagation channel behaviour for different environments, degrees of shadowing and elevation angles," IEEE Trans. Broadcasting, vol. 44, no. 1, pp. 40-76, Mar. 1998.

[21] F. Fontan, M. Vazquez-Castro, C. Cabado, J. Garcia, and E. Kubista, "Statistical modeling of the LMS channel," IEEE Trans. Veh. Technol., vol. 50, no. 6, pp. 1549-1567, Nov. 2001.

[22] A. Abdi, W. Lau, M.-S. Alouini, and M. Kaveh, "A new simple model for land mobile satellite channels: first- and second-order statistics," IEEE Trans. Wireless Commun., vol. 2, no. 3, pp. 519-528, May 2003.

[23] I. S. Gradshteyn and I. M. Ryzhik, Table of Integrals, Series, and Products. Academic Press, 2007.

[24] M. K. Simon and M.-S. Alouini, Digital Communication over Fading Channels. Wiley, 2005.

[25] A. Annamalai and C. Tellambura, "Error rates for nakagami-m fading multichannel reception of binary and M-ary signals," IEEE Trans. Commun., vol. 49, no. 1, pp. 58-68, Jan. 2001.

[26] H. Exton, Multiple Hypergeometric Functions and Applications. Wiley, 1976.

[27] N. C. Beaulieu and J. Hu, "A closed-form expression for the outage probability of decode-and-forward relaying in dissimilar Rayleigh fading channels," IEEE Commun. Lett., vol. 10, no. 12, pp. 813-815, Dec. 2006.

[28] L. Zheng and D. Tse, "Diversity and multiplexing: a fundamental tradeoff in multiple-antenna channels," IEEE Trans. Inf. Theory, vol. 49, no. 5, pp. 1073-1096, May 2003.

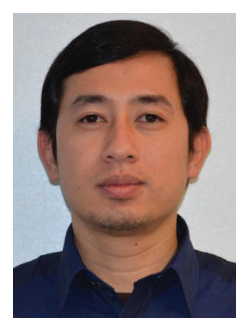

Sokchenda Sreng received the Engineer degree in Electrical Engineering from Institut de Technologie du Cambodge, Phnom Penh, Cambodia, in 2004, his M.Sc. degree in 2005, and his Ph.D. degree in 2012, both in Telecommunications and Networking from Institut National Polytechnique de Toulouse, Toulouse, France. From 2005 to 2009, he was with the department of Electrical Engineering, Institut de Technologie du Cambodge, where he was a lecturer. From 2009 to 2012, he worked as a Ph.D. student at Institut de Recherche en Informatique de Toulouse, Toulouse, France. He is currently an Assistant Professor at the department of Electrical Engineering, Institut de Technologie du Cambodge. His research interests include wireless communications, satellite communications, and cooperative communications.

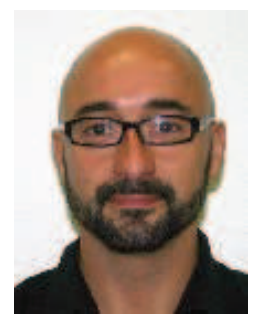

Benoît Escrig M'09) received the Engineer degree in electrical engineering from Ecole Nationale Suprieure d'Electronique, Informatique, Telecommunications, Mathematique et Mecanique de Bordeaux (ENSEIRB), Bordeaux, France, in 1992, his M.Sc. and Ph.D. degrees in signal processing from National Polytechnic Institute of Toulouse, France, in 1993 and 1997. From 1993 to 1997, he was with Rockwell-Collins France Inc, where he worked as a research engineer in High Frequency (HF) modems. He joined ENSEIRB-MATMECA, Bordeaux, France, in 1999 where he is currently an Associate Professor of Digital Communications and Communication Systems. His research interests are in wireless communications, space communications, and cooperative networks.

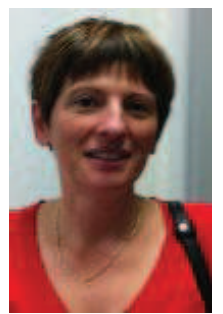

Marie-Laure Boucheret received the Engineer degree in Electrical Engineering from ENST Bretagne, Brest, France, and the M.Sc. degree in Signal Processing from the University of Rennes, both in June 1985. In june 1997, she received the Ph.D. degree in Digital Communications from TELECOM ParisTech, and the "Habilitation diriger les recherches" in June 1999 from INPT University of Toulouse. From 1985 to 1986 she has been a research engineer at the French Philips Research Laboratory (LEP). From 1986 to 1991, she has been an engineer at Thales Alenia Space, first as a project Engineer (TELECOM II program) then as a study engineer at the transmission laboratory. From 1991 to 2005 she was an Associated Professor then a Professor at TELECOM ParisTech. Since March 2005 Marie-Laure Boucheret is a Professor at the National Polytechnic Institute of Toulouse (ENSEEIHT - University of Toulouse). She is also with the Signal and Communication group of the IRIT Laboratory. Her fields of interest nclude signal processing for communications, digital receivers and satellite communications system design. 